\title{
The Robin Inequality On Certain Numbers
}

\author{
Frank Vega
}

Abstract. In mathematics, the Riemann hypothesis is a conjecture that the Riemann zeta function has its zeros only at the negative even integers and complex numbers with real part $\frac{1}{2}$. In 1915, Ramanujan proved that under the assumption of the Riemann hypothesis, the inequality $\sigma(n)<e^{\gamma} \times n \times \log \log n$ holds for all sufficiently large $n$, where $\sigma(n)$ is the sum-of-divisors function and $\gamma \approx 0.57721$ is the Euler-Mascheroni constant. In 1984, Guy Robin proved that the inequality is true for all $n>5040$ if and only if the Riemann hypothesis is true. Since then, this is called as the Robin inequality. It is known that the Robin inequality is satisfied for many classes of numbers. We show more classes of numbers for which the Robin inequality is always satisfied.

\section{Introduction}

As usual $\sigma(n)$ is the sum-of-divisors function of $n$ [1]:

$$
\sum_{d \mid n} d .
$$

Define $f(n)$ to be $\frac{\sigma(n)}{n}$. Say Robins $(n)$ holds provided

$$
f(n)<e^{\gamma} \times \log \log n .
$$

The constant $\gamma$ is the Euler-Mascheroni constant, and log is the natural logarithm. The importance of this property is:

Theorem 1.1 Robins $(n)$ holds for all $n>5040$ if and only if the Riemann hypothesis is true [3].

It is known that Robins $(n)$ holds for many classes of numbers $n$. We recall that an integer $n$ is said to be square free if for every prime divisor $q$ of $n$ we have $q^{2} \nmid n$ [1]. Robins $(n)$ holds for all $n>5040$ that are square free [1]. Let core $(n)$ denotes the square free kernel of a natural number $n$ [1]. In addition, we show that:

Theorem 1.2 Let $\frac{\pi^{2}}{6} \times \log \log \operatorname{core}(n) \leq \log \log n$ for some $n>5040$. Then Robins $(n)$ holds.

We finally prove that:

2010 Mathematics Subject Classification: Primary 11M26; Secondary 11A41, 11A25.

Keywords: Riemann hypothesis, Robin inequality, sum-of-divisors function, prime numbers. 
Theorem 1.3 Suppose that $n>5040$. Then Robins $(n)$ holds if every prime $q$ that divides $n$ satisfies

$$
q<\sqrt[\beta]{\log n}
$$

Here, it is $\beta=e^{\gamma}$.

\subsection{Known Results}

We use that the following are known:

Lemma $1.4 \quad[1]$.

$$
f(n)<\prod_{q \mid n} \frac{q}{q-1} .
$$

Lemma 1.5 [2].

$$
\prod_{k=1}^{\infty} \frac{1}{1-\frac{1}{q_{k}^{2}}}=\zeta(2)=\frac{\pi^{2}}{6}
$$

\subsection{Constants}

Let $\alpha=\frac{\pi^{2}}{6}$ and $\beta=e^{\gamma}$ where $\gamma$ is the Euler-Mascheroni constant. These constants play a key role in the next proofs.

Lemma 1.6 The following hold:

$$
\begin{aligned}
\gamma & =0.5772 \ldots \\
\alpha & =1.6449 \ldots \\
\beta & =1.7810 \ldots \\
\frac{1}{\alpha}-\frac{1}{\beta} & =0.0464 \ldots
\end{aligned}
$$

\section{A Central Lemma}

The following is a key lemma. It gives an upper bound on $f(n)$ that holds for all $n$. The bound is too weak to prove $\operatorname{Robins}(n)$ directly, but is critical because it holds for all $n$. Further the bound only uses the primes that divide $n$ and not how many times they divide $n$. This is a key insight.

Lemma 2.1 Let $n>1$ and let all its prime divisors be $q_{1}<\cdots<q_{m}$. Then,

$$
f(n)<\frac{\pi^{2}}{6} \times \prod_{i=1}^{m} \frac{q_{i}+1}{q_{i}} .
$$


Proof We use that lemma 1.4:

$$
f(n)<\prod_{i=1}^{m} \frac{q_{i}}{q_{i}-1}
$$

Now for $q>1$,

$$
\frac{1}{1-\frac{1}{q^{2}}}=\frac{q^{2}}{q^{2}-1}
$$

So

$$
\begin{aligned}
\frac{1}{1-\frac{1}{q^{2}}} \times \frac{q+1}{q} & =\frac{q^{2}}{q^{2}-1} \times \frac{q+1}{q} \\
& =\frac{q}{q-1} .
\end{aligned}
$$

Then by lemma 1.5,

$$
\prod_{k=1}^{m} \frac{1}{1-\frac{1}{q_{k}^{2}}}<\zeta(2)=\frac{\pi^{2}}{6} .
$$

Putting this together yields the proof:

$$
\begin{aligned}
f(n) & <\prod_{i=1}^{m} \frac{q_{i}}{q_{i}-1} \\
& \leq \prod_{i=1}^{m} \frac{1}{1-\frac{1}{q_{i}^{2}}} \times \frac{q_{i}+1}{q_{i}} \\
& <\frac{\pi^{2}}{6} \times \prod_{i=1}^{m} \frac{q_{i}+1}{q_{i}} .
\end{aligned}
$$

\section{Fixed Values of $\operatorname{core}(n)$}

Lemma 3.1 Robins $(n)$ holds for all $n>5040$ when core $(n) \in\{2,3,5,6,10,15,30\}$.

Proof Let $n>5040$. Specifically, let core $(n)$ be the product of the primes $q_{1}, \ldots, q_{m}$, such that $\left\{q_{1}, \ldots, q_{m}\right\} \subseteq\{2,3,5\}$. We need to prove that

$$
f(n)<e^{\gamma} \times \log \log n
$$

that is true when

$$
\prod_{i=1}^{m} \frac{q_{i}}{q_{i}-1} \leq e^{\gamma} \times \log \log n
$$

is true, because of lemma 1.4. Then, we have that

$$
\prod_{i=1}^{m} \frac{q_{i}}{q_{i}-1} \leq \frac{2 \times 3 \times 5}{1 \times 2 \times 4}=3.75<e^{\gamma} \times \log \log (5040) \approx 3.81 .
$$

However, for $n>5040$

$$
e^{\gamma} \times \log \log (5040)<e^{\gamma} \times \log \log n
$$


and hence, the proof is finished.

\section{Main Insight}

The next theorem is a main insight. It extends the class of $n$ so that Robins $(n)$ holds. The key is that the class on $n$ depend on how close $n$ is to core $(n)$. The usual classes of such $n$ are defined by their prime structure not by an inequality. This is perhaps one of the main insights.

Theorem 4.1 Let $\frac{\pi^{2}}{6} \times \log \log \operatorname{core}(n) \leq \log \log n$ for some $n>5040$. Then $\operatorname{Robins}(n)$ holds.

Proof Let $n^{\prime}=\operatorname{core}(n)$. Let $n^{\prime}$ be the product of the distinct primes $q_{1}, \ldots, q_{m}$. By assumption we have that

$$
\frac{\pi^{2}}{6} \times \log \log n^{\prime} \leq \log \log n
$$

For all square free $n^{\prime} \leq 5040$, Robins $\left(n^{\prime}\right)$ holds if and only if $n^{\prime} \notin\{2,3,5,6,10,30\}$ [1]. However, Robins $(n)$ holds for all $n>5040$ when core $(n) \in\{2,3,5,6,10,15,30\}$ due to the lemma 3.1. When $n^{\prime}>5040$, we know that Robins $\left(n^{\prime}\right)$ holds and so

$$
f\left(n^{\prime}\right)<e^{\gamma} \times \log \log n^{\prime}
$$

By previous lemma 2.1

$$
f(n)<\frac{\pi^{2}}{6} \times \prod_{i=1}^{m} \frac{q_{i}+1}{q_{i}} .
$$

Suppose by way of contradiction that $\operatorname{Robins}(n)$ fails. Then

$$
f(n) \geq e^{\gamma} \times \log \log n .
$$

We claim that

$$
\frac{\pi^{2}}{6} \times \prod_{i=1}^{m} \frac{q_{i}+1}{q_{i}}>e^{\gamma} \times \log \log n .
$$

Since otherwise we would have a contradiction. This shows that

$$
\frac{\pi^{2}}{6} \times \prod_{i=1}^{m} \frac{q_{i}+1}{q_{i}}>\frac{\pi^{2}}{6} \times e^{\gamma} \times \log \log n^{\prime} .
$$

Thus

$$
\prod_{i=1}^{m} \frac{q_{i}+1}{q_{i}}>e^{\gamma} \times \log \log n^{\prime}
$$

and

$$
\prod_{i=1}^{m} \frac{q_{i}+1}{q_{i}}>f\left(n^{\prime}\right)
$$

This is a contradiction since $f\left(n^{\prime}\right)$ is equal to

$$
\frac{\left(q_{1}+1\right) \times \cdots \times\left(q_{m}+1\right)}{q_{1} \times \cdots \times q_{m}} .
$$




\section{Implications of Main Insight}

The next lemma is a consequence of the theorem 4.1.

Lemma 5.1 Let $n>5040$ and let $n^{\prime}=\operatorname{core}(n)$. Also let $n^{\prime}$ be the product of the primes $q_{1}<\cdots<q_{m}$. Then

$$
\alpha \times \log \log n^{\prime}>\log \log n \Longrightarrow q_{m}^{\beta} \geq \log n
$$

where $\alpha=\frac{\pi^{2}}{6}$ and $\beta=e^{\gamma}$.

Proof Suppose by way of contradiction that

$$
q_{m}<(\log n)^{\frac{1}{\beta}} .
$$

We have that $\alpha \times \log \log n^{\prime}>\log \log n$ holds and so does

$$
\log n^{\prime}>(\log n)^{\frac{1}{\alpha}} \text {. }
$$

We need some basic results from number theory. As usual define $\theta(x)$ as

$$
\sum_{q \leq x} \log (q)
$$

where the sum is over all primes $q$. Note that

$$
\theta\left(q_{m}\right)=\log q_{1}+\cdots+\log q_{m} .
$$

The function $\theta(x)$ is $x$ with small error, but we will need that for $x>0$ it follows that

$$
\theta(x)<c \times x
$$

where $c=1.01624[4]$.

We note that $\theta\left(q_{m}\right)$ is greater than or equal to the logarithm of $n^{\prime}$ and so by assumptions

$$
\theta\left(q_{m}\right) \geq \log n^{\prime}>(\log n)^{\frac{1}{\alpha}}
$$

This shows that

$$
c \times(\log n)^{\frac{1}{\beta}}>c \times q_{m}>(\log n)^{\frac{1}{\alpha}} .
$$

Thus

$$
c>(\log n)^{\frac{1}{\alpha}-\frac{1}{\beta}}>(\log 5040)^{\frac{1}{\alpha}-\frac{1}{\beta}} \approx 1.10454 .
$$

This shows that we have a contradiction since $\frac{1}{\alpha}-\frac{1}{\beta}>0.0464$.

\section{Proof of Main Theorem}

Theorem 6.1 Suppose that $n>5040$. Then Robins $(n)$ holds if every prime $q$ that divides $n$ satisfies

$$
q<\sqrt[\beta]{\log n}
$$

Here, it is $\beta=e^{\gamma}$. 
Proof Let $n>5040$. There are two cases:

Case I: In this case

$$
\frac{\pi^{2}}{6} \times \log \log \operatorname{core}(n) \leq \log \log n .
$$

This case follows from theorem 4.1, $\operatorname{since} \operatorname{Robins}(n)$ is true in this case.

Case II: In this case

$$
\frac{\pi^{2}}{6} \times \log \log \operatorname{core}(n)>\log \log n .
$$

By lemma 5.1 we see that

$$
q^{\beta} \geq \log n
$$

This proves the theorem.

\section{Acknowledgments}

I thank Richard J. Lipton for helpful comments.

\section{References}

[1] YoungJu Choie, Nicolas Lichiardopol, Pieter Moree, and Patrick Solé. On Robin's criterion for the Riemann hypothesis. Journal de Théorie des Nombres de Bordeaux, 19(2):357-372, 2007. doi:10.5802/jtnb. 591.

[2] Harold M. Edwards. Riemann's Zeta Function. Dover Publications, 2001.

[3] Guy Robin. Grandes valeurs de la fonction somme des diviseurs et hypothèse de Riemann. J. Math. pures appl, 63(2):187-213, 1984.

[4] J. Barkley Rosser and Lowell Schoenfeld. Approximate Formulas for Some Functions of Prime Numbers. Illinois Journal of Mathematics, 6(1):64-94, 1962. doi:10.1215/ijm/1255631807.

CopSonic, 1471 Route de Saint-Nauphary 82000 Montauban, France Email: vega.frank@gmail.com 